\title{
A Wireless Mouse Design and Implementation Based on J2ME
}

\author{
Yuhe Song \\ College of Instrumentation \& Electrical Engineering, \\ Jilin University, Changchun 130022, China \\ songyh11@mails.jlu.edu.cn,
}

\author{
Changying Liu \\ College of Instrumentation \& Electrical Engineering, \\ Jilin University, Changchun 130022, China
}

\author{
Tianhao Wang \\ State Key Laboratory of Automobile \\ Simulation and Control, Jilin \\ University, Changchun 130025, China
}

\author{
Xin Zhang \\ College of Communication \\ Engineering, Jilin University, \\ Changchun 130012, China
}

\author{
Lingling Zheng \\ State Key Laboratory of Automobile \\ Simulation and Control, Jilin \\ University, Changchun 130022, China
}

\begin{abstract}
J2ME is a Java technology specification which was advanced by The United States of America SUN company.It was used to develop Java applications which run on small equipments, consumer electronic equipment, Internet mobile equipment, embedded equipment. Hereby, this paper will introduce a J2ME-Based program of wireless mouse; the program mainly uses Bluetooth technology and J2ME programming technology to achieve.
\end{abstract}

Keywords-J2ME; Bluetooth; Wireless mouse

\section{INTRODUCTION}

With the development of the 3G, the popularity of mobile phones has reached an unprecedented level; it is no longer a single communication device, but intelligent terminal which is the convergence of Internet and personal information processing, mobile office, entertainment, games and so on ${ }^{[6]}$.

Accurate to say that Sun Company introduced J2ME language to reduce the threshold of embedded programming, and made J2ME to become the most popular programming platform for mobile.

This paper uses the platform and combines with Bluetooth technology to draw up wireless mouse control software for a cell phone.

After two parts of the software were installed in mobile phones and personal computers, The user can use the phone to control the computer mouse remotely and to achieve the functions that mouse up, down, left, right, left-click, right-click and control the speed of mouse's movement by Bluetooth ${ }^{[1-5]}$.

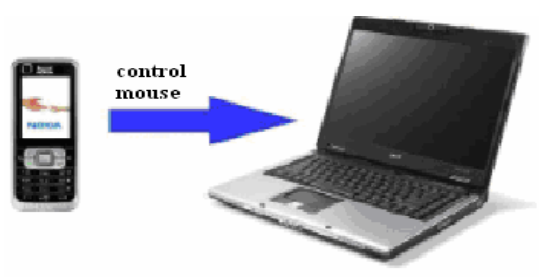

Figure 1. Vivid present

\section{J2ME-STRUCTURE SYSTEM}

The architecture of J2ME application consists of four parts: Operating System, Java Virtual Machine, CLDC/CDC and MIDP Application.

KVM (kilo virtual machine) is a part of the minimum run-time environment, it is included in the J2ME platform, applied to devices witch limited memory and CPU capacity. KVM is only an application for CLDC (Connected Limited Device Configuration); it is used to run Java software on mobile devices.

CLDC (Connected Limited Device Configuration) and CDC (Connected Device Configuration) are two confi- gurations in J2ME currently. Profile is based on Configuration, together form a complete operating environment. It classifies its equipment horizontally, and subdivides market for specific areas; its contest includes class libraries and API for special purpose ${ }^{[9]}$.

\begin{tabular}{|c|}
\hline $\begin{array}{c}\text { Profile } \\
\text { (MIDP Application) }\end{array}$ \\
\hline $\begin{array}{c}\text { CLDC/CDC } \\
\text { Libraries }\end{array}$ \\
\hline $\begin{array}{c}\text { KVM } \\
\text { (K Virtual Machine) }\end{array}$ \\
\hline $\begin{array}{c}\text { Operating System } \\
\text { (Windows,Linux) }\end{array}$ \\
\hline
\end{tabular}

Figure 2. Structure list

\section{REALIZATION OF SOFTWARE}

\section{A. The core technology}

The software achieves that the mobile phone communicate with PC through Bluetooth module. Firstly calculate the aspect ratio of the cell phone screen and computer screen, then capture and track the coordinates of the points of the computer screen. If you want to move right one pixel, then the coordinates plus one, the mouse which is written by the program correspond to the right in a pixel on the computer screen ${ }^{[6-9]}$. 
As a result, through J2ME network programming techniques, you can use the phone to control a computer mouse, and then achieve the function of wireless mouse.

\section{B. The flow chart of software}

The follow is the flow chart of software for the mobile terminal.

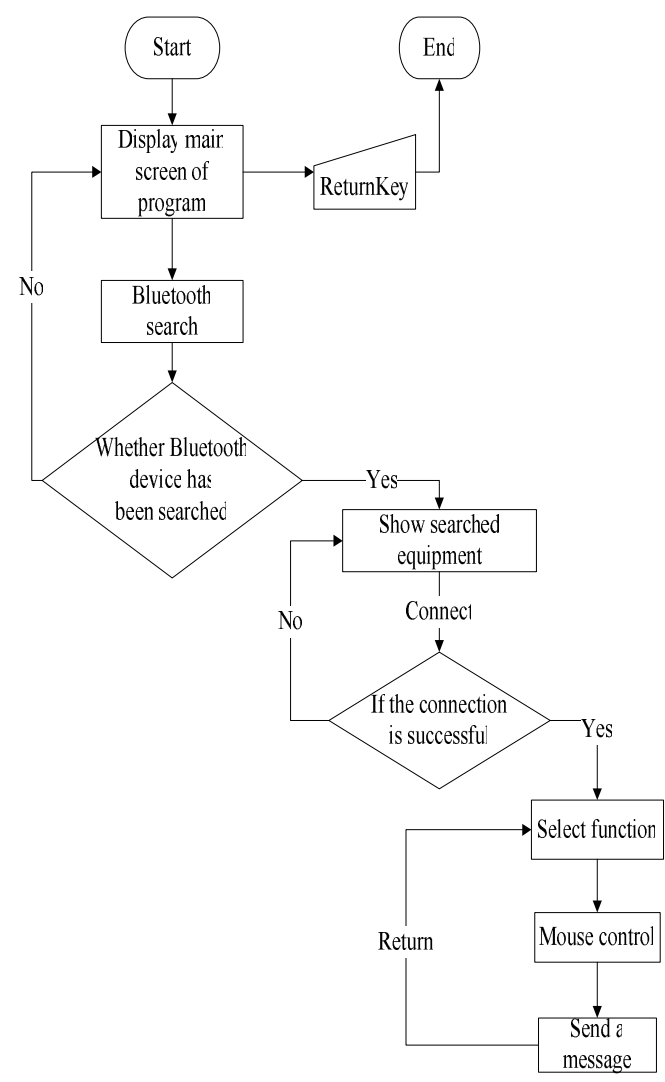

Figure 3. Flow chart for the mobile terminal.

Control module of the mobile terminal for mouse function is also achieved in REMOTE_DEVICE_SERVICE class; this class is mainly used to send mouse control information. When software for the computer terminal receives the corresponding information of mouse control, it will generate the appropriate action, such as mouse movements and mouse clicks.

List the mouse left-click on the code ${ }^{[1-5]}$ :

Public void mouse_left_kick_down () \{

Try \{

dos.writeChars ("mouse_left_kick_down");

dos.flush ();

\}

Catch (Exception e) \{

\}

\}

Public void mouse_left_kick_up () \{

Try \{

dos.writeChars ("mouse_left_kick_up");

dos.flush ();

\}

Catch (Exception e) \{

\}

\}

When the method of mouse_left_kick_down () is called,
It sends the information that the left mouse button is pressed.

When the method of mouse_left_kick_up () is called,

It sends the information that the left mouse button is released.

The follow is the flow chart of software for PC terminal $^{[10]}$.

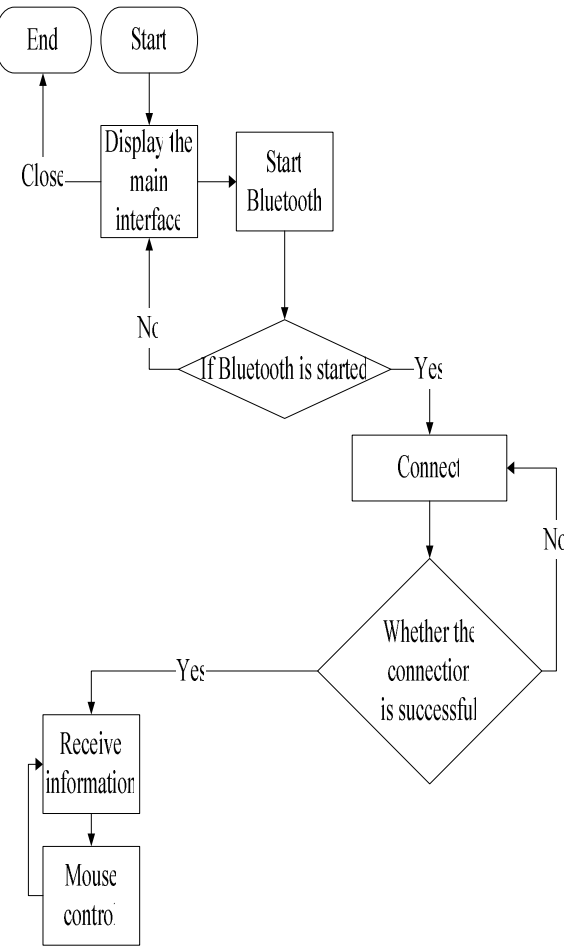

Figure 4. Flow chart for PC terminal

Control module of PC terminal for mouse function is also achieved in CLANYA_PC class. Next this paper has listed the part of program code of mouse up, mouse down and left mouse button pressed and released ${ }^{[1-5]}$.

Void mouse_up (void) \{

$/ /$ move mouse up one pixel

POINT lpPoint;

:: GetCursorPos (\&lpPoint);

:: SetCursorPos (lpPoint.x, lpPoint.y-1); return;

\} void mouse_left_kick_down (void) \{

//The left button of mouse is pressed

::mouse_event (MOUSEEVENTF_LEFTDOWN,0,0,0,0); return;

\}

Void mouse_left_kick_up (void) \{

//The left button of mouse is released

::mouse_event (MOUSEEVENTF_LEFTUP,0,0,0,0); return;

\}

The feature of mouse control module is that making some mouse action under the system scheduling module received mouse control information in code comment.

\section{Result of Software Testing}

The wireless mouse software has been tested and can be a good move up and down, left-click right-click functions. These screenshots of debugging of simulator are Figure 5 
and Figure 6.

This paper mainly designs the display interface module. Opening the software first will display the starting screen, meanwhile querying the remote Bluetooth device in the background.

After 3 seconds, display the main interface.

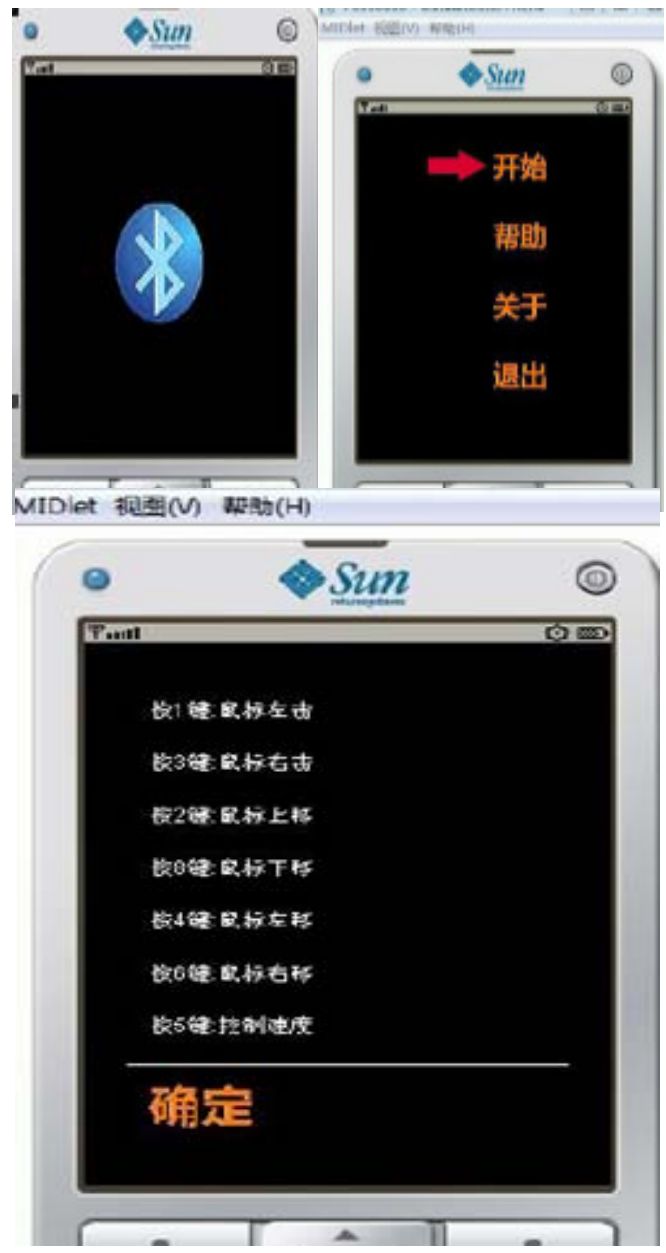

Figure 5. The working sketch of phone

The duration of starting screen is not certain, mainly to see the speed of system's operation. The main interface of software will be displayed at the end of the starting screen.

Press the 1st button, mouse left-click;

Press the 3rd button, mouse right-click;

Press the 2nd button, mouse move up; Press the 8th button, mouse move down; Press the 4th button, mouse left shift;

Press the 6th button, mouse right shift;

Press the 5th button, controlling speed;

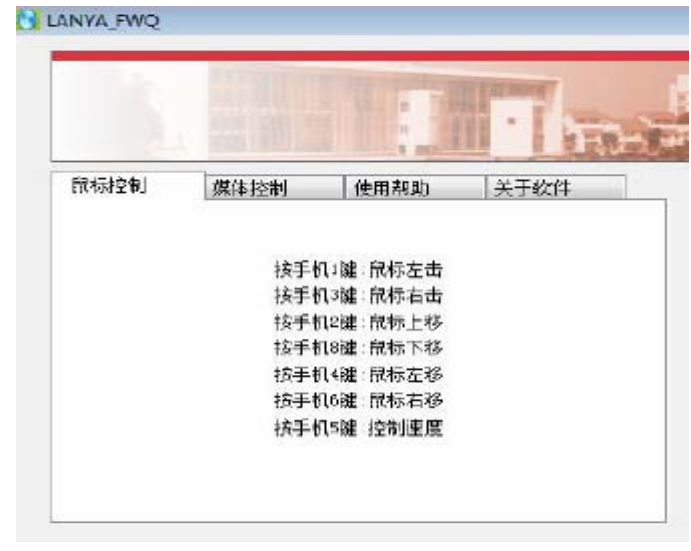

Figure 6. The working sketch of PC

The author repeatedly tested the software many times, it did not appear insensitive and no response, in line with the required functions, achieving the mouse control.

\section{CONCLUSIONS}

The software on the hardware requirements are not very high, only need a mobile phone which supports Java, a Bluetooth-enabled computers. So, the portability of the system is very strong.

With the development of mobile communication technology and the wide application of mobile device, the phone's functions will also be more powerful, and the mobile phone software which is based on J2ME technology will also become a hot topic of many mobile phone developers.

The prospect of mobile software development is prosperity. Therefore, the applicable prospects of the system are considerable, especially in the original software on the basis of improvements to upgrade, you can add music player, video, man-machine dialogue and more features. Because time and effort is not enough, this article does not involve these parts, it is an inadequacy of this article.

\section{REFERENCES}

[1]. Bin Liu JAVA ME Practical Detailed explanation-Mobile development in ECLIPSE 2007

[2]. Using Java Bluetooth wireless communication technology API "Motorola SUN mobile applications, a new bright spot Challenge" The internal data of Bluetooth networking development 2006

[3]. Liangbin Shao, Baiping Li, Xinhai Wang VisualC++.NET Programming Tsinghua University Press 2007

[4]. MSDN Microsoft Visual stdio.NET IDE integration

[5]. Chunhui Liu Study notes of Visual C++ Programming Electronic Industry Press 2008

[6]. Wisdom consulting Chinese mobile phone client software Market Analysis and Forecast Report in 2012-2016

[7]. The quick start tutorials of NetBeans_IDE_Java Internet

[8]. Xuefeng Liu, Baojie Shan Bluetooth and its applications "International Electronic Elements" 2002 The sixth period

[9]. Jianfei Zhan J2ME development Precision Solution/Java technology large Department Electronic Industry Press 2006

[10]. Chunhui Liu Visual C++ the study notes of program design Electronic Industry Press 